\title{
PHONONS IN AMORPHOUS MATERIALS
}

\section{S. Hunklinger}

Max-Planck-Institut für Festkörperforschung, Heisenbergstr. 1,7000 Stuttgard 80, F.R.G.

Résumé.- L'étude de la propagation des phonons dans les solides amorphes est un outil important pour examiner les propriétés dynamiques, parfois inattendues et surprenantes, de ces materiaux. Tout d'abord, nous présenterons et analyserons les propriétés acoustiques de la silice vitreuse, choisie comme un exemple typique. Dans la seconde partie de cette revue, nous discuterons en détail les propriétés anormales des matériaux amorphes à basse température.

Abstract. - The study of the propagation of phonons is an important tool to investigate the sometimes unexpected and puzzling dynamic properties of amorphous solids. At first, as a typical example, we shall discuss the acoustic properties of vitreous silica. In the second part of the review emphasis will be given to the anomalous low temperature properties of amorphous materials.

A. Introduction. - The vibrational excitations in crystals are generaliy well understood. In perfect crystals the vibrational motion of undamped atoms is easily decomposed into independent normal modes. These modes are plane-wave excitations, called phonons, and are characterized by a frequeney, wavevector and polarization vector. Anharmonic effects lead to a coupling between these modes resulting in a finite Iifetime. In general crystal imperfections give rise to localized excitations which can also limit the phonon lifetime through elastic or inelastic scattering.

In contrast, the situation is much more complex and much less understood in non-crystalline solids. The enormous simplification engendered by the periodicity of the crystalline structure is lacking. The proper normal modes are no longer plane-waves. One immediate consequence is that phonons are damped as in anharmonic crystals. This effect becomes more and more important as the phonon wavelength approaches the interatomic spacing. Therefore, the dispersion curve is smeared out more and more with increasing wavevector and phonons in the original sense do not exist any more. At low frequencies, i.e. in the long wavelength limit, however, glasses, like crystals, behave as elastic continua. A well defined dispersion relation exists, ana both longitudinal and transverse sound waves have been directly observed in glasses up to frequencies of about $40 \mathrm{GHz}$ by Brillouin scattering experiments. Most of the information on the vibrational properties of amorphous solids has been obtained at wavevectors close to the origin of the "Brillouin zone". There the main difference between the properties of crystals and disordered solids lies in the fact that in the latter, selection rules which are based on the long range ordex are violated. As. a consequence many effects which show sharp features in crystals, are considerably broadened in the amorphous solid.

In this review we restrict ourselves mainly to the propagation of long wavelength phonons. In the first part of this paper we will describe the propagation of phonons in vitreous silica. In the second part of the review emphasis will be placed on the anomalous low temperature properties of glasses in general.

B. Phonon Scattering in Solids. - Like the atoms of an ideal gas, the phonons in a perfect harmonic dielectric crystal are free of interaction. Thus in such a sample a sound wave would not be attenuated. In fact at low temperatures high quality dielectric crystals approach this ideal behaviour. Similar properties are expected for amorphous dielectrics because long wave-length phonons are not sensitive to the undexlying structure. However, we will see, that amorphous networks exhibit internal degrees of freedom which give rise to absorption processes much stronger than those observed in their crystalline counterparts.

Neglecting a few exceptions, the mechanisms of phonon scattering may be divided into three categories: elastic scattering, resonant absorption, and relaxation absorption. 
Scattering from inhomogenetics as, for example from frozen-in density fluctuations in glasses, belong to the first category. This process is often called Rayleigh scattering and is especially effective at short wavelengths. It contributes to the thernal resistivity of glasses at moderate temperatures ( $T \simeq 10 \mathrm{~K}$ ). For ultrasonic waves this process leads to a frequency-dependent but temperature-independent background attenuation, which can in general be neglected.

The second process is resonant absorption (direct process or one-phonon process). There, phonons are resonantly absorbed if their energy, hw, happens to coincide with the energy splitting of resonators present in the material. Very often impurities give rise to such resonating states. A well-known example is a paramagnetic ion whose ground state is split by the crystalline electric field. In the simplest case of resonators with only two states ("Two-Level Systems") we may write for the absorption $\alpha$ or the inverse mean free path $\ell^{-1}$ :

$$
\alpha_{r e s}=\frac{\pi \mathrm{NM}^{2}}{\rho \mathrm{v}^{3}} \omega \tanh \frac{\hbar \omega}{2 \mathrm{kT}}
$$

where $\mathbb{N}$ is the number of interacting systems, $\rho$ the mass density and $v$ the sound velocity. M represents the deformation potential which is given by the strength of the coupling between the resonator and the strain field of the sound wave. The factor tanh hw/2kT describes the temperature dependence which reflects the difference in the thermal population of the two states. This factor immediately makes it clear why this process is only observable for high frequencies and/or low temperatures. For $\not \omega \ll \mathrm{kT}$ the two states become equally populated and the net absorption vanishes. As we still see, the resonant interaction plays a prominent role in amorphous solids at very low temperatures.

The third process is the relaxation process which can be observed at practically all frequencies. It originates from the modulation of the thermodynamic equilibrium of a subsystem in the sample by the strain or stress field of the sound wave. Wellknown examples are electrons in metals or thermal phonons in dielectrics whose Fermisurface or frequency distribution, respectively, is modulated by the density variation associated with a longitudinal wave. The perturbed subsystem tries to $r e l a x$ into the new equilibrium via the exchange of energy. Because of the delayed response of the relaxing system, entropy is increased and the sound wave is attenuated. This relaxation can easily be visualized in the case when abubystem undergoes a structural rearrangement under the influence of the strain field. For simplicity we assume that the relaxing systems have only two possible configurational states. In this case the motion of a particle in a double-well potential (see Fig. 1) is used for the formal description. Of course, the separation between the two wells represents a generalized coordinate. It does not necessarily have the dimension of length, but can equally well be an angle or any other coordinate. The strain field, e, shifts the position of the two wells with respect to each other and varies the asymmetry, $\Delta$, by an amount $\Delta \delta=D \cdot e$, where $D$ is the deformation potential. $D$ and $M$ are related, but are not identical. M causes transitions between two states (induced dipole moment in the case of electric coupling), whereas D leads only to a shift of the two states with respect to each other (static dipole moment). Because of the variation of $\Delta$, the population of the two wells is no longer in thermal equilibrium. The systems try to reestablish thermal equilibrium via transitions of the particle from one well to the

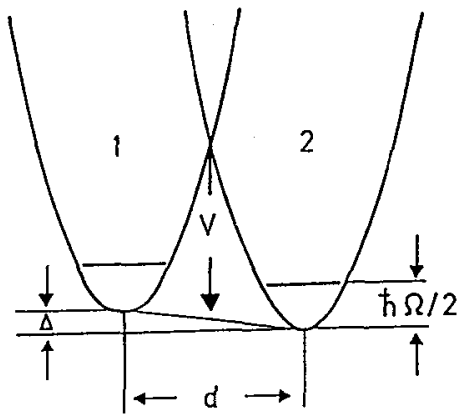

Fig. 1: Double well potential with barrier height $V$, asymmetry $\Delta$, and distance d between the two minima. $\hbar \Omega / 2$ is the ground state energy. 
other. If $\Delta \ll k T$ the following formula holds:

$$
\alpha_{r e l}=\frac{N D^{2}}{4 \rho v^{3} k T} \frac{\omega^{2} \tau}{1+\omega \omega^{2} \tau^{2}}
$$

Here $\tau$ is the time the relaxing subsystem needs to reach equilibrium again. If wT is varied, the loss goes through a maximum at $\omega \tau=1$. For $\omega \tau \gg 1$ one obtains $\alpha \propto \omega^{0} \tau^{-1}$, and for the other Iimit $\omega \tau \ll 1 \alpha \propto \omega^{2} \tau$. The quantity $\omega \tau$ can be varied either by a variation of $\omega$ or, more often, by a variation of the temperature which results in a change of $\tau$.

C. Acoustic Properties of Vitreous Silica

1. Absorption. - In Fig. 2 the attenuation of vitreous silica is plotted as a function of temperature. One experiment has been carried out at $1.5 \mathrm{MHz} / 1 /$ whereas the other curves have been obtained at $10 \mathrm{~Hz} / 2 /$. These experiments show three remarkable effects: Firstly, in all cases the attenuation increases strongly as the glass transition temperature is approached. Secondiy, although the applied frequency differs by five orders of magnitude, the absorption shows a relatively small increase with frequency. Thirdiy, differing impurity content can change the magnitude of the absorption by an order of magnitude.

This rise in the absorption close to the glass transition temperature, $T$, is a general phenomenon and is observed in other glasses as well. Since $\npreceq \omega / \mathrm{kT} \simeq \mathrm{g}_{10}{ }_{12}$ for the low-frequency data, resonant absorption can be ruled out. At the low temperature side of a relaxation peak the condition $\omega \tau$ $>1$ holds, and the observed absorption is expected to be proportional to $\omega^{0} \tau^{-1}$. Although no systematic studies of the frequency dependence at this high temperature are reported, we might infer from the data shown here and additional measurements at $37 \mathrm{kHz} / 3 /$ that the absorption depends only weakly on frequency. After subtracting the temperature independent background one obtains $\alpha \propto \exp (-Q / \mathrm{kT})$, where $Q / \mathrm{k}$ lies between 10000 and $20000 \mathrm{~K}$. Since $\alpha \propto \tau^{-1}$ this temperature dependence reflects the temperature dependence of the relaxation time. Close to $T$ the viscosity $n$ is proportional to $\tau$ so that we obtain $\eta \propto \exp Q / \mathrm{kT}$. In genera 9 the viscosity of glass at $\mathrm{T}$ follows the Vogel-FulcherTammann law $/ 4 \%$. In vitreous silica this law reduces tg a pure exponential relationship. For Suprasil the activation energy of the viscosity is of the same order of magnitude, namely about $60000 \mathrm{~K}$. Without going into details, we want to mention that the activation energy deduced from measurements close to $T$ depends on the time scale of the measurement. Q increases with increasing time ${ }^{E}$ f measurement. Although the absorption of Infrasil and Suprasil have been measured at the same frequency, the magnitude and temperature dependence of their absorption differ considerably. The main difference between these two glasses lies in their content of OH-impurities: roughly 1000 pm in the case of Suprasil whereas Infrasil is practically free of oH. Water in vitreous silica breaks up Si-0-bonds and lowers the viscosity $/ 5 /$. Simultaneously the relaxation time of the network is reduced $(n \propto \tau)$ and the absorption

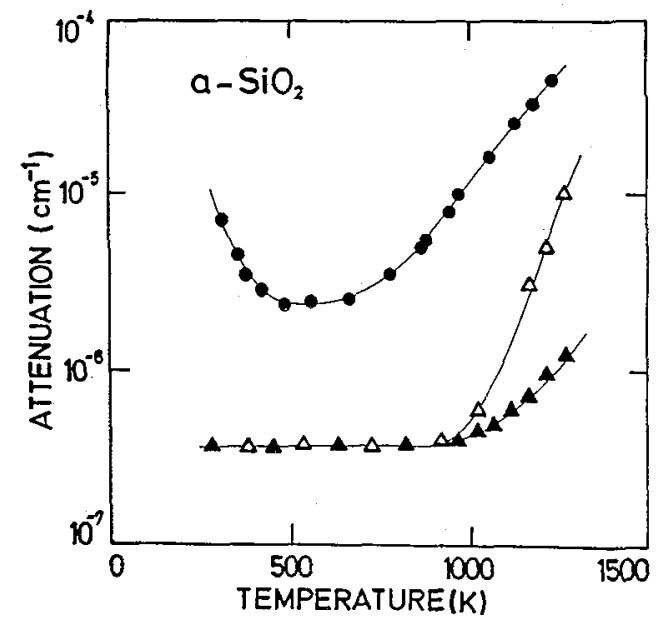

Fig. 2: Acoustic attenuation in vitreous silica as a function of temperature. - Low-OH-Suprasil (1.5 MHz, from $/ 1 /$ ), $\Delta$ Suprasi工 (10 Hz, from /2/), Infrasil $(10 \mathrm{~Hz}$, from $/ 2 /)$. 
rises with the $\mathrm{OH}$-content. The temperature independent background between room temperature and $1000 \mathrm{~K}$ is probably due to finite sensitivity of the measurement. However, experiments in the MHz range are suitable to investigate the attenuation at moderately high temperatures. Low-OH-Suprasil has been measured at $1.5 \mathrm{MHz}$; a minimum is found whose exact position depends on the heat treatment of the sample. The minimum value of the absorption is close to that of the crystalline counterpart. There the absorption is due to the interaction between the sound wave and thermal phonons. This process can be described as the high temperature limit of a relaxation process and is consequently proportional to $\omega^{2} / 6 /$. Experiments carried out at much higher frequencies ( $35 \mathrm{GHz}$ ) by Brillouin scattering $/ 7 /$, show absorption values considerabIy higher than that deduced from the MHz-measurements by extrapolation with $\omega^{2}$ law. From this result one may conclude that an additional attenuation process must be present at frequencies well above $1 \mathrm{GHz}$. The nature of this process is, however, still unknown $/ 8 /$.

The low temperature behaviour is shown in Fig. 3. In the vicinity of $50 \mathrm{~K}$ a strong absorption peak is found, caused by a relaxation process /9/. Similar absorption maxima occur in many other glasses around liquid-nitrogen temperature $/ 12 /$.

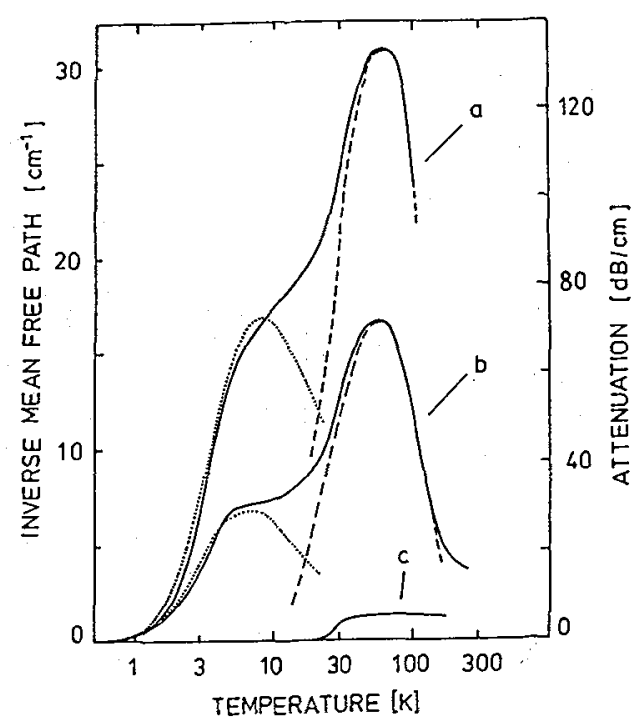

Fig. 3: Attenuation of longitudinal sound waves in vitreous silica and in a quartz crystal (from /10/). Curve a and $b$ : vitreous silica at $930 \mathrm{MHz}$ and $507 \mathrm{MHz}$ (data from /11/) curve c: crystalline quartz at $1 \mathrm{GHz}$ (data from /6/). The dashed Iines represent theoretical approximations.

In vitreous silica, measurements in a frequency range of more than six orders of magnitude indicate that this relaxation process is thermally activated and that the Arrhenius relationship can be used.

$$
\tau=\tau_{0} \exp (\mathrm{V} / \mathrm{KT})
$$

Here $\tau_{0}$ is a constant of the order of $10^{-13} \mathrm{~s}$ in vitreous silica, a value typical for lattice vibrations. $V$ is the activation energy for the relaxation process. The width of the peak is, however, much wider than expected from Eq. 2 and 3. For a better description one has to assume a distribution of $V$. A fairly good approximation is obtained, using a Gaussian distribution with its maximum at an energy corresponding to $570 \mathrm{~K}$ and a wiath of $400 \mathrm{~K} / 12 /$. It is worth mentioning that a similax loss peak has also been observed in dielectric measurements $/ 12 /$.

A number of microscopic models have been proposed to explain this relaxation process in pure, simple glasses. The common assumption is the existence of localized structural defects which may exist in two different configurations separated by a potential barrier. For vitreous silica it was proposed that a fraction of the oxygen atoms are located in a double-well potential and can move from one weil to the other 
via thermal activation. Possible defect configurations are schematically shown in Fig. 4 The oxygen atoms can move either perpendicular /13/ or parallel /14/ to the bond direction. Another possibility is the rotation of $\mathrm{SiO}_{4}$ tetrahedrons by a small. angle in a double-well potential $/ 15 \%$. These models are equally plausible for other tetrahedrically-bonded materials and similar ideas can also be developed for glasses of different structure or even for polymers.

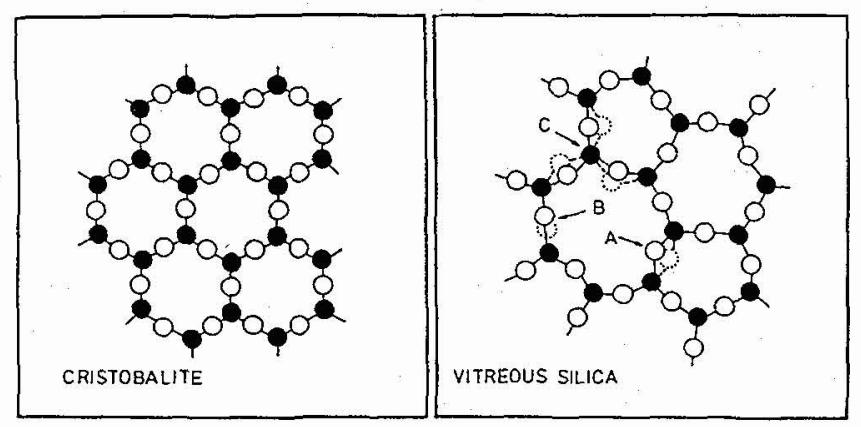

Fig. 4: Schematic two-dimensional representation of the structure of eristobalite and of vitreous silica (from /10/). Full circles represent silicon atoms and open circles oxygen atoms. Three possible types of defeets are indicated by arrows.

2. Sound Velocity in Vitreous Silica. - Absorption processes always cause a variation of the velocity of sound $v$ that can be calculated via the Kramers-Kronig relation. In contrast to the weak negative temperature coefficient in pure crystals which is mainly due to anharmonicities, much stronger variations, both positive and negative, are found in glasses. In Fig. 5 the variation in vitreous silica is plotted for temperatures up to $1300 \mathrm{~K}$. This measurement was carried out at $35 \mathrm{GHz}$ using Brillouin scattering $/ 7,16 /$. At the lowest temperatures shown in this plot, the velocity decreases linearly with temperature. Qualitatively this variation can be understood as a consequence of the absorption process taking place in this temperature range. It is, however, worth mentioning that the linearity of the decrease cannot be explained by current theories. Around $50 \mathrm{~K}$ a minimum in $\mathrm{v}$ is observed and up to $500 \mathrm{~K} v$ rises linearly again. Above this temperature the increase becomes less

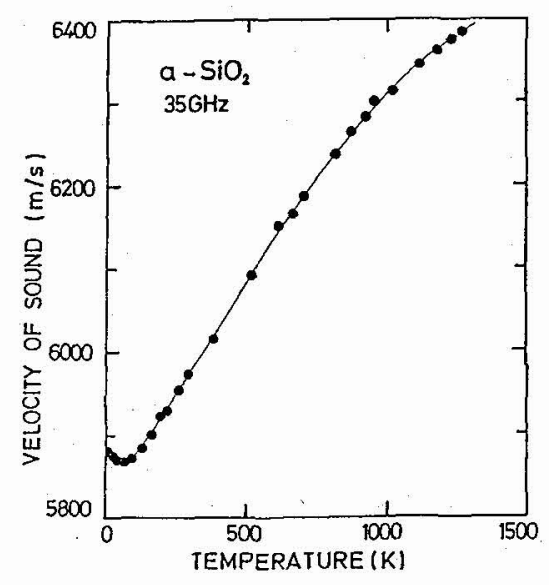

Fig. 5: Velocity of longitudinal sound waves of $35 \mathrm{GHz}$ in vitreous silica measured by Brillouin scattering (from $/ 7,16 /$ ).

and less steep and goes through a maximum at around $1500 \mathrm{~K} / 17 /$. The increase of $\mathrm{v}$ with temperature is not yet understood although several mechanisms have been proposed. At present it seems that it is a pecularity of tetrahedrically coordinated glasses $/ 18 /$. The magnitude of the temperature coefficient is expected to increase with the bond angle at the oxygen atom (or other bridging atom). 
D. Low-Temperature Properties of Amorphous Solids. - During the last teri years there have been intense efforts to understand the anomalous-low-temperature properties of glasses. These anomalies are unexpected for the following reason. At low temperatures the thermal behaviour of pure dielectric solids should be determined by acoustic phonons of long wavelength. Below $1 \mathrm{~K}$ the wavelength of the corresponding dominant phonons is larger than $1000 \AA$ and therefore much larger than the scale of microscopic disorder. As a consequence, at such temperatures there should be hardly any difference between the thermal properties of amorphous and crystalline dielectrics. Although there is great regularity in the thermal properties of amorphous materials, their behaviour, contrary to expectation, is completely different from that of pure erystaline substances. This contradictory aspect has stimulated not oniy extensive work on thermal properties but also a new sequence of quite different investigations on the mechanical and dielectric properties of glasses below $1 \mathrm{~K}$.

1. Specific Heat of Glasses at Low Temperatures. - As mentioned above, the low temperature specific heat of pure dielectric solids should be determined by long wavelength phonons. According to Debye's theory, the specific heat below a few Kelvin should be proportional to $\mathrm{T}^{3}$. Glasses show, however, an unexpected linear term in the specific heat below $1 \mathrm{~K} / 19 /$. This behavjour is shown in Fig. 6 where the specific heat of vitreous silica and crystalline quartz are plotted. At a temperature of $25 \mathrm{mK}$ the measured specific heat of vitreous silica /20/ exceeds the Debye contribution of the phonons by a factor of 1000! Similar discrepancies were found to exist

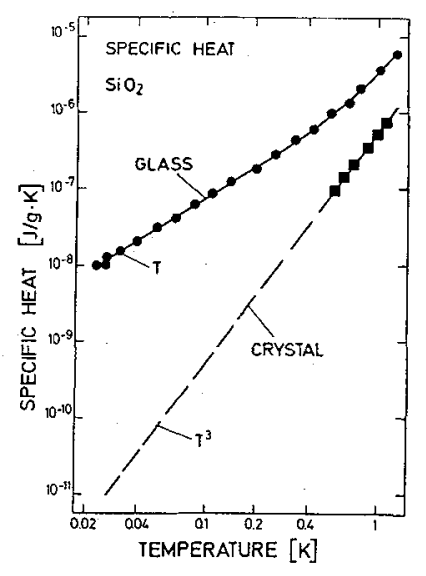

Fig. 6: Specific heat of vitreous silica and erystalline quartz as a function of temperature (from /19/ and $/ 20 /)$. The specific heat of $a$ pure quartz crystal is proportional to $\mathrm{T}^{3}$ between $0.6 \mathrm{~K}$ and $5 \mathrm{~K}$. Below $0.6 \mathrm{~K}$ no experimental data exist so far.

for all other amorphous insulators so far studied, including amorphous polymers $/ 21 /$. Also glassy metals exhibit this anomaly. Recently this has been demonstrated by measurements of the specific heat of amorphous superconductors well below the transition temperature where the electronic specific heat can be neglected /22/. From this result one may conclude that electrons do not contribute to the extra specific heat but instead atomic motions are responsible in amorphous insulators as well as in amorphous metals. In this context it is interesting to note that a linear specific heat has also been found in crystalline ionic conductors or quenched single crystals demonstrating that this anomalous themal properties are an even more general phenomenon $/ 23 /$.

This large extra specific heat clearly demonstrates the existence of a new type of Low-energy state which is specific to the disordered state and does not exist in pure crystals. Surprisingly not only the temperature dependence of this extra specific hat similar for all amorphous materials but also its magnitude varies only within an order of magnitude for different glasses. Typicaliy these disordered solids contain roughly $10^{-17} \mathrm{~cm}$ low-energy excitations uniformly distributed in the energy interval from 0 to $1 \mathrm{~K}$. The total number of excitation is, however, probably considerably higher, since the density of states extends to higher energies and seems to increase quadratically /21/. Unfortunately no reliable estimate of this number 
can be given since at higher temperatures the extra specific heat is affected by the contribution of phonons whose contribution cannot be calculated due to the lack of proper information on the phonon dispersion relation. Most of these low-energy excitations interact, as we will see, strongly with the amorphous network, i.e. the phonons. But for an appreciable number, the interaction time with the "lattice" is much longer than $1 \mu \mathrm{s}$ and extends to at least $10^{4} \mathrm{~s}$. Consequently the specific heat depends on the time of measurement. Successful observations of such a time dependent heat capacity have recently been reported by several groups /24/.

2. Ultrasonic Properties of Insulating Glasses. - Attenuation /25/ as well as velocity /26/ of an ultrasonic wave are strongly affected by the interaction with these low-energy excitations. As a typical example the attenuation as a function of temperature is shown in Fig. $7.127 \%$. Let us first focus our attention to temperatures below. $1 \mathrm{~K}$ where a characteristic dependence on the acoustic intensity is observed. At. higher intensities the ultrasonic loss vanishes with decreasing temperature; at very low power levels, however, absorption inereases again. Obviously such behaviour is not compatible with a loss caused by a relaxation process, which is intensity independent. It can be explained by the resonant absorption of strongly anharmonic oscillators. A more detailed analysis of the data shows that they are best described by the resonant transition between two energy levels: At higher acoustic intensities both levels reach equal population because of the finite lifetime in the excited state (of the order of $10 \mu$ in our example) and consequently the absorption falls. This leads to the observed intensity dependence and we have to replace Eq. 1 by

$$
\alpha_{\text {res }}=\frac{\pi n_{0} M^{2}}{\rho v^{3}} \omega\left(1+J+J_{c}\right)^{-1 / 2} \tanh \frac{\hbar \omega}{2 k T}
$$

where $n$ is the constant density of states of the "two-level systems" (TLS), $J$ is the acoustic input intensity and $J$ is the critical value around which the saturation of the upper level becomes noticeable. In fact, the temperature dependence of the absorption $\alpha_{\mathrm{res}}$ at low intensities $/ 28 /$ and its intensity dependence /29/. strongly support the assumption that TLS and not multi-level systems are present in glasses.

These TIS also cause the steep rise of the attenuation at higher temperatures $130 /$. The sound wave modulates the level splitting of the TIS and leads to a relaxation process as described by Eq. 2. The TLS relax with a relaxation time, $\tau$, given by

$$
\tau^{-1}=\left(\frac{M_{1}^{2}}{v_{I}^{5}}+\frac{2 M_{t}^{2}}{v_{t}^{5}}\right) \quad \frac{E^{3}}{2 \pi \rho \pi^{4}} \operatorname{coth} \frac{E}{2 k T}
$$

where $M$ is the deformation potential, already introduced in $E q .1$ and the indices 1 and $t$ refer to longitudinal and transverse waves, respectively. Because of the distribution of the energy splitting we have to replace the number of relaxing systems $N$

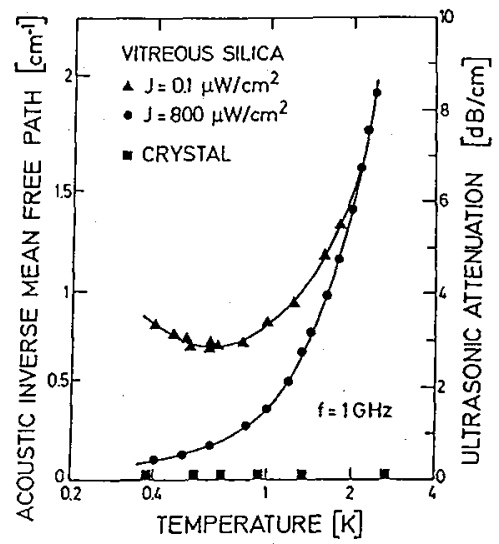

Fig. 7: Temperature dependence of the attenuation of longitudinal acoustic waves of two different intensities in vitreous silica. The full squares indicate the temperature independent attenuation in a pure quartz crystal (from /27/). 
by an integral over this distribution. According to Eq. $2 \alpha \propto \tau^{-1}$ if $\omega \tau>1$ holds. In silicate glasses this condition is fulfilled for frequencies around $1 \mathrm{GHz}$ up to temperatures of a few Kelvin. Since the energy splitting of the dominant systems is proportional to $\mathrm{T}$ we expect $\alpha \propto \mathrm{T}^{3}$. This prediction has indeed been observed $131 /$ and demonstrates once more the dominant role of the TIS. In summary the ultrasonic and hypersonic absorption in glasses at low temperatures is caused by the interaction of the sound wave with a broad distribution of low-energy excitations which are best described by TLS.

3. Velocity of Sound. - Since the ultrasonic absorption depends on temperature the same must be true for the velocity of sound, because both quantities are linked by the Kramers-Kronig relation. Fig. 8 shows the temperature dependence of the velocity of sound in vitreous silica for longitudinal sound waves./27/. The velocity increases due to the resonant interaction, passes a maximum, and decreases because of the predominance of the relaxation process. Applying the Kramers-Kronig relation to Eq. 4 and integrating over the energy distribution of the TLS one obtains for the variation of the velocity of sound $\Delta v=v(T)-v\left(T_{0}\right)$, where $T_{0}$ is an arbitrary reference temperature:

$$
\left(\frac{\Delta v}{v}\right)_{r e s}=\frac{n_{0} M^{2}}{\rho v^{2}} \ln \frac{T}{T_{0}}
$$

From the slope of the curve at the lowest temperatures one can directly determine the product of the density of states of the TLS and the coupling strength. This result is important in the discussion of the thermal conductivity. Interestingly the velocity of sound or the corresponding elastic stiffness of glasses decreases on cooling in contrast to that of crystalline materials which generally show no variation or the opposite behaviour at temperatures below $1 \mathrm{~K}$.

For completeness we want to mention that analogous anomalies are observed for the dielectric behaviour of glasses /12/. For example the dielectric absorption can be saturated at higher electric field strength and the dielectric constant varies logarithmically at low temperatures. The magnitude of these effects, however, in contrast to the acoustic properties, depends strongly on the impurity content. These observations demonstrate that TLS carry not only an elastic but also and electric dipole moment.

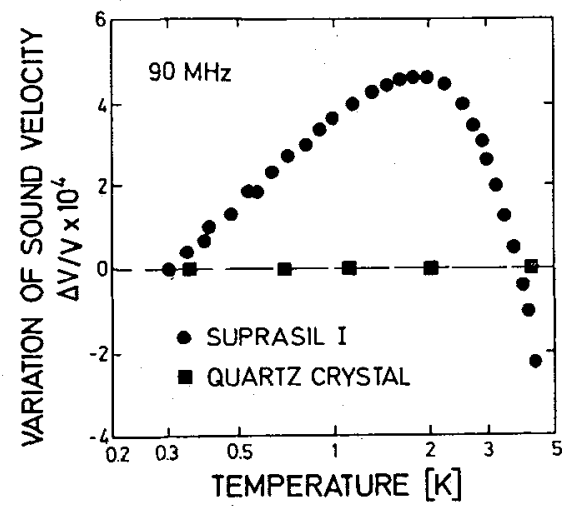

Fig. 8: Relative variation of longitudinal sound velocity in vitreous silica as a function of temperature (from /26/). Reference temperature $\mathrm{T}_{0}=0.3 \mathrm{~K}$. Full squares show for comparison the independent velocity in a quartz crystal.

4. Thermal Conductivity of Insulating Glasses. - In glasses ultrasonic and hypersonic phonons are mainly scattered resonantly by TLS. For thermal phonons the same process is dominant and the thermal conductivity of glasses is limited to values much lower than that of the corresponding crystalline material. Since the TLS are localized, only Debye phonons take part in heat conduction. Therefore it is proportional to the product of the phonon heat capacity $\left(\propto \mathrm{T}^{3}\right)$ and the phonon mean free path, l. At_frequencies corresponding to thermal energies $\omega \simeq \mathrm{k} T / h$ we obtain from $\mathrm{Eq}$. i $\alpha \propto 2^{-1} \propto \mathrm{T}$. Consequently the thermal conductivity of glasses should vary as 
$\mathrm{T}^{2}$ in agreement with experiment /19-23/. Interestingly, the strength of the coupling between acoustic waves and TLS is of the same order of magnitude in all silicate and chalcogenide glasses. Consequently the heat conductivity of all these substances is almost identical below $1 \mathrm{~K}$. Even glassy polymers and the lattice contribution of metallic glasses /32,33/ behave in the same way.

5. Hole Burining and Coherent Excitation of TLS. - So far we have discussed the interaction of the TLS with external acoustic fields. But an interaction also exists among the TLS which is probably elastic in origin. This additional aspect is taken into account by using the Bloch-equations in order to describe the dynamical bahaviour of the TLS $/ 34,35 /$. In analogy to paramagnetic ions in a magnetic field we have to distinguish between $\mathrm{T}_{1}$ and $\mathrm{T}$, the longltudinal and transverse relaxation times, respectively. $\mathrm{T}$ is the spontâneous lifetime caused by the emission or absorption of thermal phonons and is identical to the relaxation time we have discussed so far. $\mathrm{T}_{2}$ is determined by the interaction between the TLS.

In principle both quantities can be measured by applying more than one acoustic or electric pulse to the sample. Here we discuss briefly the so-called "hole-burning" experiment $136 /$. When a relatively strong ultrasonic pulse propagates through a glass sample at low temperatures, one would expect a saturation only of those TLS whose energy splitting is sufficiently close to the energy of the incident phonons. If, however, the degree of saturation is actually measured using a second weak probing pulse of different frequency, saturation is still observed even if the frequencies of both pulses differ by as much as $\pm 50 \mathrm{MHz}$. Since the lifetime broadening of the 'L'LS at the measuring temperature of $0.5 \mathrm{~K}$ and the frequency uncertainity of the sound pulse accounts only for roughly $1 \mathrm{MHz}$, the result is rather surprising. The results of such a measurement are shown in Fig. 9 for two different times of observation $137 /$. Clearly, the width of the hole also depends on time. This large spectral width arises from pressure fluctuations at the site of the resonant TLS caused by neighbouring 'I'LS due to absorption and reemission of thermal phonons. These fluctuations lead to a modulation of the energy splitting of the resonant TLS and hence to a temperature dependent line width, since the number of thermally activated TIS increases with temperature. Because of the statistical character of the interaction between the resonating system and its neighbour, i.e. the extent of time correlation, the line width also depends on time $/ 38 /$.

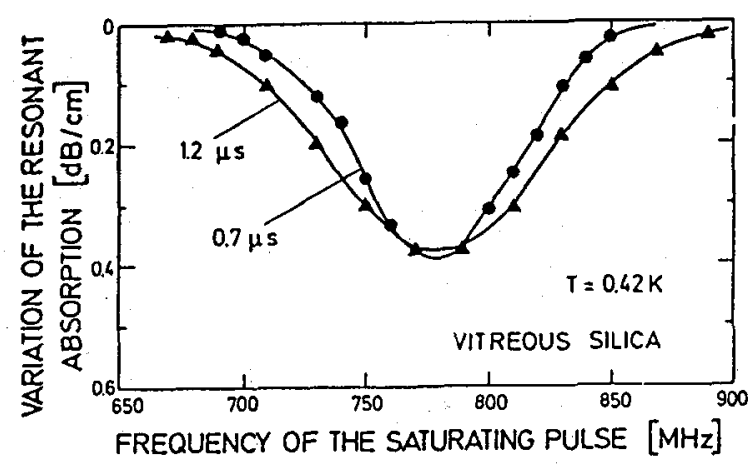

Fig. 9: Attenuation of a weak probing pulse of fixed frequency $(780 \mathrm{MHz})$ as a function of the frequency of the intense saturating pulse for two different pulse durations (from /37/).

On cooling the sample to the Millikelvin range the relaxation times $T_{1}$ and $T_{2}$ become larger and larger and finally exceed the duration of applied acoustic pulses? Under this condition the TLS can be excited coherently, $i . e$. a microscopie elastic (or electric) polarization can be generated/39/. A variety of new phenomena are based on this coherence effect. We only mention the direct measurement of the decay of the polarization ("free induction decay"), the generation of echoes (spontareous as well as stimulated echoes), population inversion and the investigation of self-induced transparency /40/. Echoes in glasses have been studied in many experiments; thus $T_{1}$ and $\mathrm{T}_{2}$ have been measured in vitreous silica down to temperatures of $4 \mathrm{mK} / 41 / \mathrm{At}$ 
$20 \mathrm{mk}$ for vitreous silica typical numbers are $140 \mu \mathrm{s}$ for $\mathrm{T}_{1}$ and $10 \mu \mathrm{s}$ for $\mathrm{T}_{2}$ of TLS having an energy splitting corresponding to $700 \mathrm{MHz}$.

6. Glassy Metals. - As already mentioned above, amorphous superconductors exhibit a Iinear specific heat well below $\mathrm{T} / 22,42 /$. Since its magnitude is comparable to that of ordinary glasses it seems that TLS are also present in amorphous metals in roughly the same numbers as in vitreous silica. Below $1 \mathrm{~K}$ the heat conduction shows the same $\mathrm{T}^{2}$-dependence /32,33/ as is known for amorphous dielectrics indicating that in amorphous metals heat is also mainly transported by phonons whose mean free path is limited by scattering from TLS. Detailed studies of the temperature variation of the sound velocity showed similar results to ordinary glasses $/ 43 /$. For the ultrasonic absorption a temperature and intensity dependence was found /44/ similar to that shown in Fig. 4 for vitreous silica.

But there is a basic difference: the saturation effect is observed at much lower temperatures and, even more important, saturation occurs at completely different acoustic intensities. While in vitreous silica the resonant absorption at $1 \mathrm{GHz}$ and $0.5 \mathrm{~K}$ becomes saturated at an intensity of $10^{-7} \mathrm{~W} / \mathrm{cm}^{2}$ one needs an intensity which is three orders of magnitude higher in PdSi at $60 \mathrm{mK} / 44 /$. This demonstrates that, in normal conducting metals, because of the presence of conduction electrons, the relaxation times are shortened by several orders of magnitude relative to ordinary glasses, where the TLS can only relax via phonons.

If the conduction electrons are condensed to Cooper-pairs by cooling a superconducting amorphous metal well below $T$, one would predict that the relaxation time should increase by several orders ${ }^{c}$ of magnitude finally reaching the value of insulating glasses $/ 45 /$. Therefore a drop in the relaxation absorption is expected at $T$ since below these temperatures the condition $\omega \tau \gg 1$ should not hold. In fact, this behaviour has been observed /46/. In Fig. 10 the attenuation of $745 \mathrm{MHz}$ shear waves is plotted as a function of temperature for $\mathrm{Cu}_{60} \mathrm{Zr}_{40}$. At $\mathrm{T}_{c}=0.4 \mathrm{~K}$ the attenuation shows the expected drop.

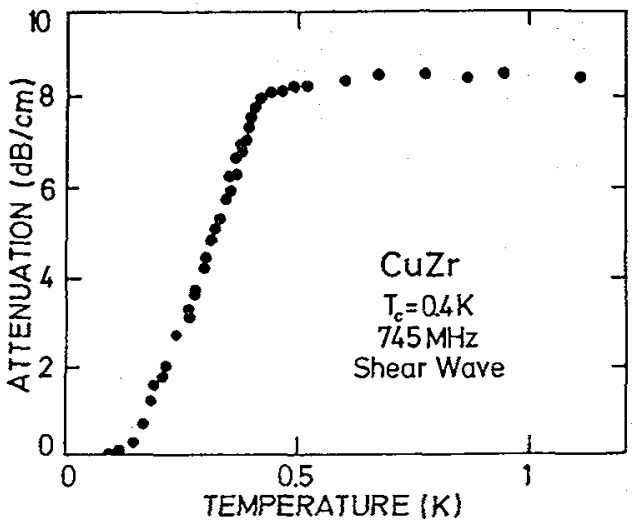

Fig. 10: Attenuation of shear waves in the superconducting glassy metal $\mathrm{Cu}_{60} \mathrm{Zr}_{40}$ Transition temperature $T_{c}=0.4 K$ (erom /46/).

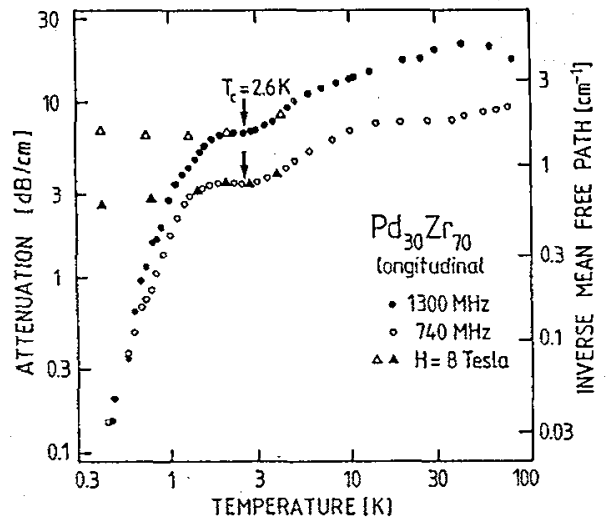

Fig. 11: Temperature dependence of the ultrasonic attenuation $\mathrm{PQ}_{30} \mathrm{Zr}_{70}$ in the superconducting and normal stale (from $/ 47 /)$.

Historically, the first acoustic measurements on a superconducting glass were carried out on $\mathrm{Pd}_{30} \mathrm{Zr}_{70}$ /48/. These data are shown in Fig. 11. Two interesting features are worth mentioning. Firstly, the application of a magnetic field of 8 Tesla makes this sample normally conducting, and the unpaired electrons give rise to a strong absorption down to the lowest temperatures. Secondly, the drop in the absorption is not observed at $\mathrm{T}$ but well below this temperature. This makes it clear that the condition $\omega T={ }_{1}{ }^{c}$ which is typical for the occurence of a relaxation 
can also be fulfilled at temperatures below $T$. This result demonstrates how effective free electrons are in reducing the relaxation time of TLS. Although the number of unpaired electrons drops rapidly below $T$ those which are left over, are still capable of shortening the relaxation time to ${ }^{c}$ such an extent that $\omega T>1$ is. not fulfilled until $T \simeq \mathrm{T} / 2$ is reached. Without going into details we want to mention that a kink in the velocity of sound is expected at $T_{c}$ and this has been observed in CuZr as well as in PdZr $/ 46,47 /$.

6. On the Nature of the Low-Energy Excitations. - The universality of these low temperature anomalies suggests that the TLS in amorphous solids are a natural consequence of the metastable state of glassy materials with many configurational minima of the free energy, which are almost but not exactly equivalent. Many of the local minima are separated by high barriers and are not accessible at low temperatures. However, a fraction of them may have a low enough barrier to permit motion at low temperatures by quantum-mechanical tunneling. It has been proposed to approximate this complicated situation by a simple model, the so-called tunneling model $/ 49 /$. The starting point is very similar to that used in the description of the loss peak observed in vitreous silica around $50 \mathrm{~K}$. It is assumed that clusters of atoms are able to undergo structure relaxation even at the lowest temperatures, and that this possibility may be described by the motion of a particle (of still unknown structure) in a double-well potential (see Fig. 1). The probability for tunneling depends on the overlap of the wavefunctions in the wells. If $\lambda=d(2 m V / R \Omega)^{1 / 2}$ is the tunneling parameter with $\mathrm{m}$ as the mass of the tunneling particle, then the tunneling motion leads to a level splitting given by $\Delta_{0}=\pi \Omega \exp (-\lambda)$. Here $\pi_{\Omega}$ is of the order of the separation of the energy levels in the isolated well. The total energy splitting of the tunneling system which is equivalent to the energy. splitting of a TLS is given by $E=\left(\Delta_{0}^{2}+\Delta^{2}\right)^{1 / 2}$. The basic assumption of the tunneling model is now that the asymmetry $^{\circ} \Delta$ and the tunneling parameter $\lambda$ are uniformly distributed, $i . e$.

$$
\mathrm{p}(\Delta, \lambda) \quad \mathrm{a} \Delta \quad \mathrm{d} \lambda=\overline{\mathrm{P}} \quad \mathrm{d} \Delta \quad \mathrm{d} \lambda
$$

where $\overline{\mathrm{P}}$ is a constant. These tunneling states couple with the outside world in a similar way to that already discussed for the classical structural defects. An external strain field, e, shifts the relative position of the two minima by an amount $\delta \Delta=\gamma \cdot e$ where $\gamma$ is the deformation potential. Since $M=\gamma \Delta / E / 35 /$, the coupling parameter introduced for the TLS depends on the tunnel splitting $\Delta_{0}$. As a consequence TLS of the same energy splitting may be coupled differently and so the relaxation given in $\mathrm{Bq}$. 5 exhibits a distribution.

Acoustic measurements offer an interesting possibility to study the validity of the distribution function ( $E q .7$ ). Measurements on the resonant process and on the relaxation process in the regime $\omega \tau>1$ are sensitive to strongly coupled. tunneling states, where $\Delta / E \simeq 1$ and $M \simeq \gamma$. If, however, those tunneling systemș which exhibit the minimum relaxation time $\tau$, are able to reach equilibrium during the period of a sound wave, then the dominamin'contribution to the absorption is due to weakiy coupled systems, for which $\Delta / \mathrm{F} \simeq 0$ and $\mathrm{M} \simeq 0$. For the regime $\omega \tau_{\text {in }}<1$ one finds

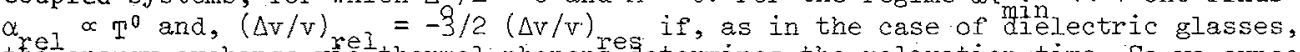
thel energy exchange vial thermal phononsegetermines the relaxation time. So we expect a plateau in the absorption at higher temperatures and logaritnmic decrease of the velocity. In dielectric glasses this behaviour is not easily observed since for utrasonic trequencies $\omega \tau_{\text {r }}=1$ is found to be at temperatures of several kelvin where additional effects already start to play an important role. In metallic glasses the plateau can be observed since $\omega \tau$. $=1$ is reached at relatively low temperatures due to the TLS-electron interacmin. A nice example has already been show in Fig. 10 for the case of CuZr, where the expected plateau is clearly visible. Until very recently the logarithmic decrease of the velocity of sound has never been observed. In Fig. 11 we show the first measurement of the absorption and the velocity that has been carried out at a frequency low enough to reach the condition wi $<1$ well below $1 \mathrm{~K} / 50 /$. The temperature variation of both quantities in a silicate glass is found to follow the prediction of the tunneling model. Therefore this measurement demonstrates that the distribution of Eq. 7 also holds for large values of the tunneling parameter $\lambda$ responsible for low relaxation rates. Above $1 K$, however, the velocity does not follow the predicted curve any more. This probably indicates that above this temperature relaxation processes other than the direct one become noticable. 
Although this phenomenological tunneling model is very successful, there are still some astonishing aspects and open questions. For example, it is worthwile to point out that the experimentally observed relaxation times extend over 13 orders of magnitude. Another intriguing aspect is the observation that ILS exist in a wide variety of different glasses and that they are always coupled to the "lattice" with nearly the same strength. What really causes the chemistry to play such a minor role? Undoubtedly, the most interesting and as yet unsolved question is the microscopic nature of the tunneling systems. On one hand they cannot involve too many atoms since otherwise the potential minima in the configuration space will be far apart and the barrier separating them will be high. On the other hand they cannot involve too few atoms since otherwise the probability of finding two nearly equivalent minima in the free energy will be small. In vitreous silica it is likely /10/ that the rotary motion of a $\mathrm{SiO}_{4}$-tetrahedron is responsible. This motion is probably made feasible by the softness of the bending motion of the bridging oxygen bonds. In other materials one can also find clusters of similar size which can rotate. Whatever the nature of the bonding is, it seems that some arrangements of atoms are needed where bonding constraints are relaxed resulting in the formation of clusters. In amorphous silicon and germanium which have to satisfy the rigid constraints of covalent bonding, cluster formation does not seem to be likely and TLS apparently do not exist ejther $/ 51 /$.

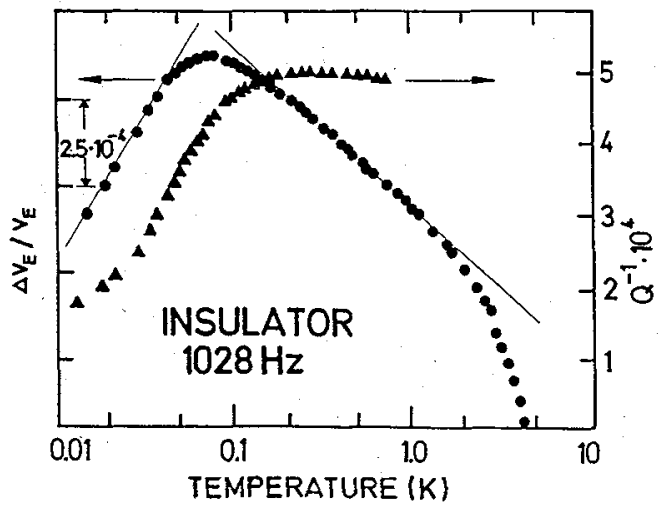

Fig. 12: Variation of the sound velocity (left scale) and internal friction $Q^{\top}=2 \alpha_{\mathrm{v}} / \omega$ (right scale) of a silica based glass. This experiment was carried out at $1028 \mathrm{~Hz}$ using a vibrating reed.

F. Summary. - In summary, we may say that in amorphous materials the propagation of phonons is fundamentaliy different from that in perfect crystals. In adition to the attenuation processes. found in crystalline materials, a relaxation process close the glass transition temperature and a structural relaxation at a much lower temperature is observed. In contrast to expectation the low temperature properties of amorphous solids also differ fundamentally from their crystalline counterparts. Although the formal phenomenological description of the observation in terms of "two-level. systems" or "tunneling states" has been very successful, we are still far from a complete understanding of the low temperature properties of amorphous solids on an atomic scale.

Acknowledgements. - The author is grateful to A. Raychaudhuri, M.v. Schickfus, and $\widehat{G .}$ Weiss for many stimulating discussions. 


\section{References}

/1/ FRASER D.B., J. Appl. Phys. 41, (1970) 6

12/ BRÜCKNER R., J. Non-Cryst. SOlids 5, (1970) 123

13 / MARX J.W., SIVERTSEN J.M., J. Appl. Phys. 24, (1953) 81

14/ See for example: GUTZOW I. in Amorphous Materials (DOUGLAS R.W., and ELLIS B., eds.) Wiley, London (1972) p. 159

15/ HETHERINGTON G., JACK K.H., KENNEDY J.C., Phys.. Chem. Glasses $\underline{5}$, (1964) 130

16/ BÖMMEL H.E., DRANSFELD K., Phys. Rev. 117, (1960) 1245

/7/ PELOUS J., VACHER R., Solid state Comm. 18, (1976) 637

181 VACHER R., PELOUS J., PLICQUE F., ZAREMBOWITCH A., J. Non-Cryst. Sol. 45, (1981) 397

19/ FINE M.E., DUYNE van H., KENNY N.T., J.Appl. Phys. 25, (1954) 402

$/ 10 /$ HUNKIINGER S., Proc. UItrasonic Symp. (IEEE), 1974, p. 443

/11/ JONES C.K., KLEMENS P.G., RAYNE J.A., Phys. Lett. 1 (1964) 31

$112 /$ See for example: HUNKLINGER S., SCHICKFUS M. von, in Topics in Current Physics (W.A. Phillips ed.) Springer, Berlin - Heidelberg 1981, Vol. 24, p. 81

/13/ ANDERSON O.L., BÖMMEL H.E., J.-Am. Ceram. Soc. 38, (1955) 125

/14/ STRRAKNA R.E., Phys. Rev. 123, (1961) 2020

/15/ VUKCEVICH M.R., Non-Cryst. Solids 11, (1972) 25

/16/ VACHER R., PELOUS J., Phys. Rev. B 14, (1976) 823

$/ 17 /$ SPINNER S., J. Am. Ceram. Soc. $45,(1962) 394$

/18/ THOMAS N., Thesis, Univercity of Cambridge (1978)

/19/ ZELIER R.C., POHL R.0., Phys. Rev. B 4, (1971) 2029

/20/ LASJAUNIAS J.C., RAVEX A., VANDORPE M.', HUNKLINGER S., Solid State Comm. 17 (1975) 1045

/21/ STEPHENS R.B., Phys. Rev. B $\underline{8},(1973) 2896$

122/ GRAEBNER J.E., GOLDING B., SCHUTZ R.J., HSU F.S.L., CHEN H.S., Phys. Rev. Lett. 39, (1977) 1480

123/ MCWHAN D.B., VARMA C.M., HSU F.L.S., REMEIKA J.P., Phys. Rev. B 15, (1977) 553; LOU L.F., Solia State Comm. 19, (1976) 335

124/LOPONEN M.T., DYNES R.C., GARNO J.P., NARAYANAMURTI H., Phys. Rev. Lett. 45 (1980) 457; MEISSNER M., SPITZMANN K., Phys. Rev. Lett. 46 (1981) 265; ZIMMERMAINN J., WEBER G., Phys. Rev. Lett. 46, (1981) 661

125/ HUNKLINGER S., ARNOLDS W., STEIN S., NAVA R., DRANSFEID K., Phys. Lett. A 42 , (1972) 263; GOLDING B., GRAEBNER J.E., HALPERIN B.I., SCHUTZ R.J., Phys. Rev. Lett. 30, (1973) 223

126/ PICHí L., MAYNARD R., HUNKLINGER S., JÄCKLE J., Phys. Rev. Lett. 32 , (1974) 1426

127/ HUNIKINGER S., in Festkörperprobleme (Adv. Solid State Phys.), (J. Treusch ed.), Vieweg, Braunschweig 1977, Vol 17, p.1

128/ GOLDING B., GRAEBNER J.E., SCHUTZ R.J., Phys, Rev. B 14, (1976) 1660

/29/ ARNOLD W., HUNKLINGER S., STEIN S., DRANSFELD K., T. Non-Cryst. SOl. 14, (1974) 192

130/ JÄCKLE J., Z. Phys. 257, (1972) 212

131/ JÄCKLE J., PICHE L., ARNOLD W., HUNKLINGER S., J. NOn-Cryst. SOI. 2O, (1976) 365

132/ MATEY J.R., ANDERSON A.C., Phys. Rev. B 16, (1977), 3406

133/ RAYCHAUDHURI A.K., HASEGAWA R., Phys. Rev. B 21, (1980) 479

134/ JOFFRIN J., LEVELUT A., J. Physique 36 , (1975) 811

135/ HUNKLINGER S., ARNOLD W., in PhysicaI Acoustics (W.P. Mason and R.N. Thurston eds.) Academic Press, New York 1976, Vol 12, p. 155

/36/ ARNOLD W., HUNKLINGER S., Solia State Comm. 17, (1975) 883

/37/ ARNOLD W., MARTINON C., HUNKLINGER S., J. Physique Lett. 39, (1978) C6-916

138/ BLACK J.L., HALPERIN B.I., Phys. Rev. B 16; (1977) 2879

139/ GOLDING B., GRAEBNER J.E., Phys. Rev. Lett. 37, (1976) 852

$140 /$ see for example: GOLDING B., GRAEBNER in Topics in Current Physics (W.A.

Phillips ed.) Springer, Berlin - Heidelberg, 1981, Vol. 24, p. 106

$141 /$ BERIVARD L., PICHE L., SCHUMACHER G., JOFFRIN J., GRAEBUER J., J. Physique Lett. 39, (1978) 126

142/ See for example:LÖHNEYSEN H. von, Phys. Reports 79, (1981) 161 
/43/ BELLESSA G., BETHOUX 0., Phys. Lett. 62A, (1977) 125; BELLESSA G., J. Phys. C, 10 (1977) L285

144/ GOLDING B., GRAEBNER J.E., KANE A.B., BLACK J.L., Phys. Rev. Lett. 41 , (1978) 1487; DOUSSINEAU P., LEGROS P., L.EVELUT A., ROBIN A., J. Physique Lett. (1978) 265

145/ BLACK J.L., FULDE P., Phys. Rev.Lett. 43 , (1979) 453

146/ ARNOLD W., BILLMANIN A., DOUISSINEAU P., LEVEIUT A., to be published in Physica (1982)

147/ WEISS G., HUNKLINGER S., LÖHNEYSEN H. von, to be published in Physica (1982)

148/ WEISS G., ARNOLD W., DRANSFELD K., GÜNTHERODT H.J., Solid state Comm. 33, (1980) 11

/49/ PHILLIPS W.A., J. Low Temp. Phys. 7, (1972) 351; ANDERSON P.W., HALPERIN B.I., VARMA C., Phil. Mag. 25, (1972) 1

/50/ RAYCHAUDEURI A.K., HUNKLINGER S., to be published in this volume

/51/ HAUMEDER M. Von, STROM U., HUNKLINGER S., Phys. Rev. Lett. 44, (1980) 84 